\title{
Stability of ferromagnetism in the half-metallic pnictides and similar compounds: A first-principles study
}

\author{
E Şaşıg̃lu†, I Galanakis $\ddagger$, L M Sandratskii $\dagger$ and P Bruno $\dagger$ \\ † Max-Planck Institut für Mikrostrukturphysik, D-06120 Halle, Germany \\ $\ddagger$ Institut of Microelectronics, NCSR "Demokritos", 15310 Aghia Paraskevi, \\ Athens, Greece \\ E-mail: ersoy@mpi-halle.de, i.galanakis@fz-juelich.de, \\ lsandr@mpi-halle.de, bruno@mpi-halle.de
}

\begin{abstract}
Based on first-principles electron structure calculations and employing the frozen-magnon approximation we study the exchange interactions in a series of transition-metal binary alloys crystallizing in the zinc-blende structure and calculate the Curie temperature within both the mean-field approximation (MFA) and random-phase approximation (RPA). We study two Cr compounds, $\mathrm{CrAs}$ and $\mathrm{CrSe}$, and four Mn compounds: $\mathrm{MnSi}, \mathrm{MnGe}, \mathrm{MnAs}$ and $\mathrm{MnC} . \mathrm{MnC}, \mathrm{MnSi}$ and $\mathrm{MnGe}$ are isovalent to $\mathrm{CrAs}$ and $\mathrm{MnAs}$ is isoelectronic with CrSe. Ferromagnetism is particular stable for $\mathrm{CrAs}$, MnSi and MnGe: All three compounds show Curie temperatures around $1000 \mathrm{~K}$. On the other hand, CrSe and MnAs show a tendency to antiferromagnetism when compressing the lattice. In $\mathrm{MnC}$ the half-metallic gap is located in the majority-spin channel contrary to the other five compounds. The large half-metallic gaps, very high Curie temperatures, the stability of the ferromagnetism with respect to the variation of the lattice parameter and a coherent growth on semiconductors make MnSi and CrAs most promising candidates for the use in spintronics devises.
\end{abstract}

PACS numbers: $75.47 . \mathrm{Np}, 75.50 . \mathrm{Cc}, 75.30 . \mathrm{Et}$

\section{Introduction}

The emerging field of spintronics [1] 2], also known as magneto- or spin-electronics, has provided a strong motivation towards the fabrication of novel magnetic materials. A strong interest has been focused on the so-called half-metallic ferromagnets, i.e. metallic compounds for which either the majority or minority spin-channel presents a gap at the Fermi level $E_{F}$ 3. Such behavior is detected in various perovskite structures [4, some oxides like $\mathrm{CrO}_{2}$ [5] and magnetite $\left(\mathrm{Fe}_{3} \mathrm{O}_{4}\right)$ [6], several Heusler alloys [7, 8, 9, or some diluted magnetic semiconductors (DMS) [10, 11].

In 2000, Akinaga and collaborators succeeded to grow a thin film of the binary alloy $\mathrm{CrAs}$ on top of GaAs [12]. It was found that $\mathrm{CrAs}$ was actually continuing the zinc-blende (zb) structure of GaAs crystallizing in a metastable phase. The most astonishing property was the total spin magnetic moment of $3 \mu_{B}$, which is consistent with the half-metallic character predicted by relevant electronic structure calculations published in the same paper and reproduced with larger accuracy in latter publications 13, 14. The thin films of CrAs have also the great advantage of a high 
Curie temperature $T_{\mathrm{C}}$, exceeding the $400 \mathrm{~K}$ (limit of the magnetometer used in the experiment) [15]. This discovery intrigued the interest of experimentalists and several attempts were made to grow similar metallic compounds as thin films. Zhao et al. have grown zb CrSb films by molecular beam epitaxy and found a $T_{\mathrm{C}}$ similar to the $\mathrm{CrAs}$ case 16. Moreover, the growth of small zb MnAs islands on GaAs substrates 17. has been achieved and $\mathrm{CrAs} / \mathrm{GaAs}$ multilayers have been fabricated [18] showing that coherent heterostructures are possible. These half-metallic materials combine high values of $T_{\mathrm{C}}$ with the coherent growth on top of semiconductors and as the knowhow on the growth increases, they can be considered as very promising candidates for spintronics applications.

Several theoretical studies have been devoted to the zinc-blende compounds of transition elements with group-V and VI elements (respectively, pnictides and chalcogenides). The calculations of the total energy and structural optimization show, in agreement with experiment, that the ground state crystal structure of these compounds is not of the zinc-blende type. In most cases the equilibrium crystal structure is of the NiAs type [19]. This structure does not lead to half-metallicity 20, 21, 22, 23, 24]. The zinc-blende structure can only be obtained by the epitaxial growth of few layers on semiconductors. Galanakis and Mavropoulos studied in detail the magnetic properties and the position of the Fermi level in zb V-, Cr- and Mn-pnictides and chalcogenides as a function of the lattice constant 25]. Several authors have confirmed these results [26, 27, 28, 29, 30, 31, 32]. Some of the binary semiconductors crystallize in the wurtzite structure and a number of studies is devoted to such systems [33 34. Of special interest is the case of half-metallic zb MnC compound whose properties differ from the properties of other Mn compounds [35]. Also the cases of unsupported clusters [36] and nanocrystallites [37 have been studied.

An important aspect of the studies is the stability of the half-metallic gap. It has been shown that the tetragonal deformation of the zb lattice does not destroy the gap [38, 39] and for some systems it even makes the zb structure more stable [40, 41]. Contrary to other half-metallic systems, in the case of the zb transition metal compounds it is possible to retain the half-metallicity both at the surfaces of these materials [25, 42] and in the case of multilayers with binary semiconductors 43, 44, 45, 46, 47, 48. The $\mathrm{V}$ and $\mathrm{Cr}$ atoms have the ability to increase considerably their spin moment as a result of decreasing number of neighbors keeping in this way the half-metallicity of the system.

Contrary to $\mathrm{Cr}$ and $\mathrm{V}$, the $\mathrm{Mn}$ spin moment is saturated in these compounds that leads to the loss of the half-metallicity at the surface [25]. The spin-orbit coupling has very weak effect on the spin-polarization of the electron states at the Fermi level for the $s p$ atoms up to 5 th period of the Mendeleev's table [49]. In the case of $\mathrm{MnBi}$, where $\mathrm{Bi}$ is a heavy element from the 6th period, the spin-orbit coupling leads to the decrease of the spin-polarization from $100 \%$ to $77 \%$ [49. Recently, Chioncel and collaborators studied the influence of the correlation effects on the electron structure of $\mathrm{CrAs}[50]$. They found that the spin-magnon interaction leads to the appearance of nonquasiparticle states in the spin-minority channel. The states are shown to lie above the Fermi level and to be sensitive to the lattice constant.

In contrast to the gap properties, the exchange interactions in these compounds attracted much less attention. Shirai has shown that in the case of pnictides (VAs, CrAs and MnAs) the ferromagnetic state is energetically preferable compared to the antiferromagnetic state [13]. Only in the case of $\mathrm{zb}$ FeAs an opposite trend was found 13. Sakuma demonstrated that the Mn compounds isoelectronic to CrAs ( $\mathrm{MnSi}$, 
MnGe and MnSn) are half-metallic ferromagnets [51. In these compounds, similar to $\mathrm{CrAs}$, the long-range ferromagnetism is stabilized by the short-range direct $\mathrm{Mn}-\mathrm{Mn}$ interaction and an indirect interaction through the $s p$ atoms. The Curie temperature in both $\mathrm{CrAs}$ and the isoelectronic MnZ compounds was found to exceed $1000 \mathrm{~K}$ [51. Kübler calculated $T_{\mathrm{C}}$ for VAs, CrAs and MnAs and found the largest value of $1041 \mathrm{~K}$ in the case of CrAs compared with $764 \mathrm{~K}$ in VAs and $671 \mathrm{~K}$ in MnAs [52]. Taking MnAs as an example, it was shown that the loss of the half-metallicity in a compressed lattice leads to a strong decrease of the Curie temperature [52]. Sanyal and collaborators have calculated $T_{\mathrm{C}}$ for $\mathrm{V}-, \mathrm{Cr}-$ and $\mathrm{MnAs}$ compounds assuming the GaAs and InAs lattice constants [28. Their mean field approximation (MFA) results agree with the calculations of other authors while the values of $T_{\mathrm{C}}$ obtained with Monte Carlo simulations are $\sim 100 \mathrm{~K}$ lower than the MFA values for VAs and MnAs and $\sim 300 \mathrm{~K}$ for CrAs. Note that the mean-field formula for the Curie temperature used in references [28, 51] is derived for a single-sublattice system. Therefore the exchange interaction between $3 d$ and $s p$ atoms is not treated consequently. Also the formula of Kübler 52 is derived for an one-sublattice case. In general, the treatment of a multiple-sublattice materials needs solving a system of equations with the number of equations equal to the number of sublattices (section 2). Below we show that for materials studied in this paper the one-sublattice approximation is well founded. In other systems, however, it leads to a large error [56, 57.

In this work we report the theoretical investigation of the magnetic exchange interactions for selected zb compounds. We study two $\mathrm{Cr}$ compounds, CrAs and $\mathrm{CrSe}$, and four Mn compounds, MnAs, MnSi, MnGe and MnC. MnAs is isoelectronic to CrSe whereas other three Mn compounds are isoelectronic to CrAs. We use the calculated exchange parameters to estimate the Curie temperature in both the mean field approximation $\left(T_{\mathrm{C}}^{\mathrm{MFA}}\right)$ and random phase approximation $\left(T_{\mathrm{C}}^{\mathrm{RPA}}\right)$. A special attention is paid to the influence of the position of the Fermi level on the magnetic properties.

The paper is organized as follows: In section 2] we discuss the calculational technique. In sections 3 and 4 we present the results for the $\mathrm{Cr}$ and $\mathrm{Mn}$ based halfmetallic compounds. Section 5 is devoted to MnC. Finally we summarize in section 6

\section{Calculational Method}

The calculations are carried out using the augmented spherical waves method (ASW) [53] within the atomic-sphere approximation (ASA) [54]. The exchange-correlation potential is chosen in the generalized gradient approximation [55]. A dense Brillouin zone (BZ) sampling $30 \times 30 \times 30$ is used. The radii of all atomic spheres are chosen equal.

The method of the calculation of the exchange constants has been already presented elsewhere [56. Here, to make the paper reasonably self-contained a brief overview is given. We describe the interatomic exchange interactions in terms of the classical Heisenberg Hamiltonian

$$
H_{\text {eff }}=-\sum_{\mu, \nu} \sum_{\substack{\mathbf{R}, \mathbf{R}^{\prime} \\\left(\mu \mathbf{R} \neq \nu \mathbf{R}^{\prime}\right)}} J_{\mathbf{R} \mathbf{R}^{\prime}}^{\mu \nu} \mathbf{s}_{\mathbf{R}}^{\mu} \mathbf{s}_{\mathbf{R}^{\prime}}^{\nu}
$$

In equation 1 the indices $\mu$ and $\nu$ number different sublattices and $\mathbf{R}$ and $\mathbf{R}^{\prime}$ are the 
lattice vectors specifying the atoms within sublattices, $\mathbf{s}_{\mathbf{R}}^{\mu}$ is the unit vector pointing in the direction of the magnetic moment at site $(\mu, \mathbf{R})$.

We employ the frozen-magnon approach [58 to calculate interatomic Heisenberg exchange parameters. The calculations involve few steps. In the first step, the exchange parameters between the atoms of a given sublattice $\mu$ are computed. The calculation is based on the evaluation of the energy of the frozen-magnon configurations defined by the following atomic polar and azimuthal angles

$$
\theta_{\mathbf{R}}^{\mu}=\theta, \quad \phi_{\mathbf{R}}^{\mu}=\mathbf{q} \cdot \mathbf{R}+\phi^{\mu} .
$$

The constant phase $\phi^{\mu}$ is always chosen equal to zero. The magnetic moments of all other sublattices are kept parallel to the $\mathrm{z}$ axis. Within the Heisenberg model (1) the energy of such configuration takes the form

$$
E^{\mu \mu}(\theta, \mathbf{q})=E_{0}^{\mu \mu}(\theta)+\sin ^{2} \theta J^{\mu \mu}(\mathbf{q})
$$

where $E_{0}^{\mu \mu}$ does not depend on $\mathbf{q}$ and the Fourier transform $J^{\mu \nu}(\mathbf{q})$ is defined by

$$
J^{\mu \nu}(\mathbf{q})=\sum_{\mathbf{R}} J_{0 \mathbf{R}}^{\mu \nu} \exp (i \mathbf{q} \cdot \mathbf{R}) .
$$

In the case of $\nu=\mu$ the sum in equation (4) does not include $\mathbf{R}=0$. Calculating $E^{\mu \mu}(\theta, \mathbf{q})$ for a regular $\mathbf{q}-$-mesh in the Brillouin zone of the crystal and performing back Fourier transformation one gets exchange parameters $J_{0 \mathbf{R}}^{\mu \mu}$ for sublattice $\mu$. The determination of the exchange interactions between the atoms of two different sublattices $\mu$ and $\nu$ is discussed in reference [56].

First, the Curie temperature is estimated within the mean-field approximation (MFA) for the case of a multi-sublattice material by solving the system of coupled equations [56, 59]

$$
\left\langle s^{\mu}\right\rangle=\frac{2}{3 k_{B} T} \sum_{\nu} J_{0}^{\mu \nu}\left\langle s^{\nu}\right\rangle
$$

where $\left\langle s^{\nu}\right\rangle$ is the average $z$ component of $\mathbf{s}_{\mathbf{R}}^{\nu}$ and $J_{0}^{\mu \nu} \equiv \sum_{\mathbf{R}} J_{0 \mathbf{R}}^{\mu \nu}$. Equation 5 can be represented in the form of eigenvalue matrix problem

$$
(\mathbf{\Theta}-T \mathbf{I}) \mathbf{S}=0
$$

where $\Theta_{\mu \nu}=\frac{2}{3 k_{B}} J_{0}^{\mu \nu}, \mathbf{I}$ is a unit matrix and $\mathbf{S}$ is the vector of $\left\langle s^{\nu}\right\rangle$. The largest eigenvalue of matrix $\Theta$ gives the value of $T_{\mathrm{C}}^{\mathrm{MFA}}$ 59.

Within the random phase approximation (RPA) the Curie temperature is given by the relation 62

$$
\frac{1}{k_{\mathrm{B}} T_{\mathrm{C}}^{\mathrm{RPA}}}=\frac{6 \mu_{\mathrm{B}}}{M} \frac{1}{N} \sum_{q} \frac{1}{\omega(\mathbf{q})},
$$

where $\omega(\mathbf{q})$ is the spin-wave dispersion. Equation (7) does not take into account the presence of several sublattices and will be used to estimate the Curie temperature with account for the interactions within the sublattice of the $3 d$ atoms. As we will show these interactions give a leading contribution to the Curie temperature. 
Table 1. Calculated atom-resolved and total spin moments in $\mu_{\mathrm{B}}$ for $\mathrm{CrAs}$ and $\mathrm{CrSe}\left(2^{\text {nd }}\right.$ to $6^{\text {th }}$ column$)$. CrAs and CrSe are half-metallic for the GaAs and CdS experimental lattice constants, respectively. $\mathrm{a}_{I I}$ means that the Fermi level is at the upper edge of the gap and $\mathrm{a}_{I I I}$ corresponds to $1 \%$ contraction with respect to $\mathrm{a}_{I I}$.

\begin{tabular}{lc|ccccc}
\hline Compound & $\mathrm{a}(\AA)$ & $m^{\mathrm{Cr}}$ & $m^{\text {As,Se }}$ & $m^{\text {Void1 }}$ & $m^{\text {Void2 }}$ & $m^{\text {Total }}$ \\
\hline $\left.\mathrm{CrAs}-\mathrm{a}_{I[\text { GaAs }}\right]$ & 5.65 & 3.24 & -0.31 & -0.03 & 0.10 & 3.00 \\
$\mathrm{CrAs}-\mathrm{a}_{I I}$ & 5.53 & 3.17 & -0.25 & -0.03 & 0.11 & 3.00 \\
$\mathrm{CrAs}-\mathrm{a}_{I I I}$ & 5.47 & 3.10 & -0.23 & -0.03 & 0.10 & 2.95 \\
\hline $\mathrm{CrSe}-\mathrm{a}_{I[C d S]}$ & 5.82 & 3.92 & -0.10 & 0.02 & 0.16 & 4.00 \\
$\mathrm{CrSe}-\mathrm{a}_{I I}$ & 5.62 & 3.84 & -0.04 & 0.02 & 0.18 & 4.00 \\
$\mathrm{CrSe}-\mathrm{a}_{I I I}$ & 5.56 & 3.77 & -0.04 & 0.02 & 0.16 & 3.92 \\
\hline
\end{tabular}

\section{Cr-compounds: CrAs and CrSe}

We begin with the discussion of the $\mathrm{Cr}$ compounds: pnictide $\mathrm{CrAs}$ and chalcogenide CrSe. The half-metallicity in these compounds results from the formation of bonding and antibonding hybrides between the $t_{2 g}$ states $\left(d_{x y}, d_{y z}\right.$, and $\left.d_{x z}\right)$ of the transition metal atom (here $\mathrm{Cr}$ ) and the $p$ states of the four neighboring $s p$ atoms (As or Se). The hybridizational gap is partly filled by the $e_{g}$ states of the $3 d$ atom $\left(d_{z^{2}}\right.$ and $\left.d_{x^{2}-y^{2}}\right)$. The hybridization takes place in both spin channels. The position of the hybridization gap is different for majority and minority electrons as a consequence of the exchange splitting [25]. There are in total four occupied minority states, one coming from the $s$ states of the $s p$ atom and three bonding $3 d$ states. The minority-spin $e_{g}$ orbitals lay above the Fermi level. Thus the total spin moment $M_{t}$ in $\mu_{\mathrm{B}}$ is given by the relation $M_{t}=\left(Z_{\mathrm{tot}}-8\right) \mu_{B}$, where $Z_{\text {tot }}$ is the total number of valence electrons in the unit cell [25]. In the cases of CrAs (11 valence electrons) and $\mathrm{CrSe}$ (12 valence electrons) the total spin moment should be 3 and $4 \mu_{B}$, respectively.

In table 1we present the atom-resolved and total spin moments. Notations Void1 and Void2 are used for two types of empty spheres used in our ASA calculations to describe correctly the charge distribution in the zinc-blende structure. For each system we performed calculations for three different lattice parameters. The first parameter (case I) corresponds to the experimental lattice constant of $\mathrm{GaAs}(\mathrm{CdS})$ for $\mathrm{CrAs}(\mathrm{CrSe})$. For these lattice constants both compounds are half-metals. This is seen in figure 1 where the spin-projected density of states (DOS) is presented. The total spin moment is exactly integer as expected for a half-metallic system. The $\mathrm{Cr}$ atoms posses a very large spin moment of $3.24 \mu_{\mathrm{B}}$ in CrAs that reaches the value of $3.92 \mu_{\mathrm{B}}$ in CrSe. A larger moment of $\mathrm{CrSe}$ is a consequence of one extra valence electron per formula unit. Both As and Se atoms have small induced moments antiparallel to the $\mathrm{Cr}$ moments. The discussion of the spin moments of the $s p$ atoms and their relative magnitude can be found in reference [25].

The half-metallic gap of CrSe is much larger than for CrAs. This is explained by the larger $\mathrm{Cr}$ moment in CrSe that leads to a larger exchange splitting $\Delta E_{x}$ and, as a result, to a higher position of the minority $e_{g}$ bands.

In the next step, we compress the lattice simulating a hydrostatic pressure. The contraction of the lattice constant results in a higher energy position of the majority $p$ states with a corresponding increase of the Fermi energy, $E_{\mathrm{F}}$. The calculations are performed for two decreased values of the lattice parameter. The first (case II) 

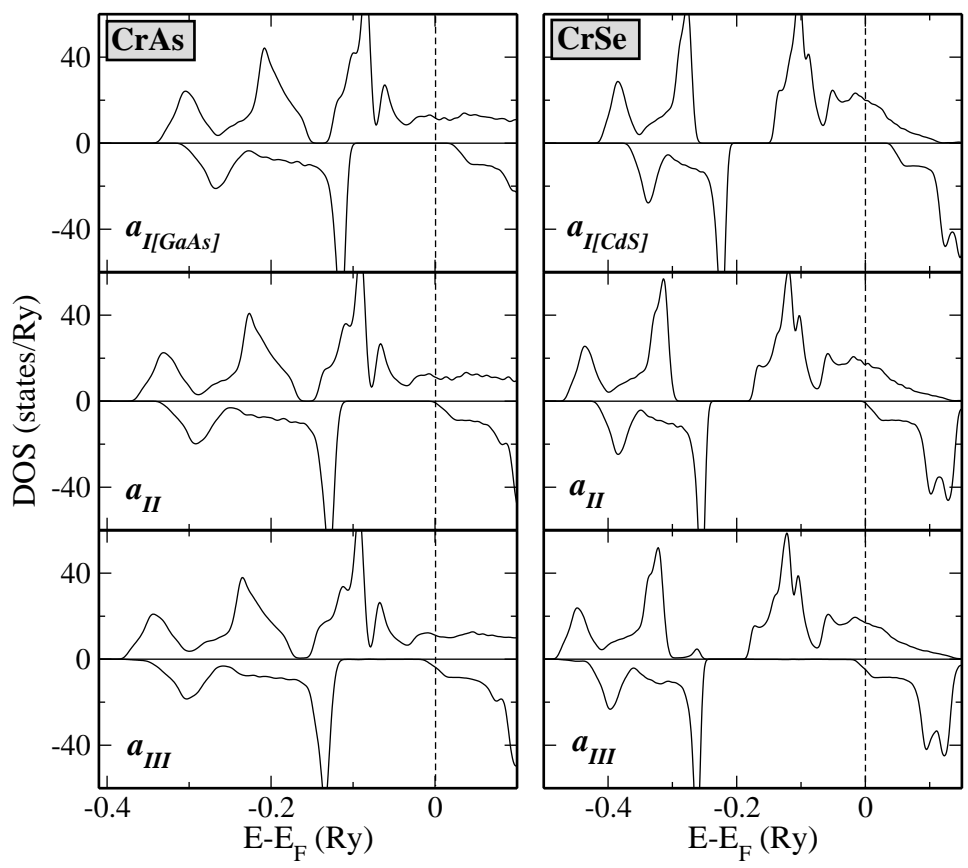

Figure 1. Calculated spin-resolved density of states of $\mathrm{CrAs}$ and $\mathrm{CrSe}$. The upper panel corresponds to the experimental lattice constant of GaAs and CdS, respectively, the middle panel to the $\mathrm{a}_{I I}$ case in table 1 (Fermi level at the higherenergy edge of the half-metallic gap) and the bottom to the $\mathrm{a}_{I I I}$ case in the same table ( $1 \%$ contraction of the lattice constant with respect to the $\mathrm{a}_{I I}$ case).

corresponds to the position of the Fermi level at the bottom edge of the minority-spin conduction band (fig. (1). The second parameter (case III) is obtained by a further $1 \%$ decrease of the lattice constant. In the latter case the Fermi level lies within the spinminority conduction band. Since half-metallicity is lost the total magnetic moment in case III becomes non-integer (table 1).

The contraction of the lattice leads to an increase of the hybridization between the $\mathrm{Cr}$ states and the states of the $s p$ atoms. As the result, both the $\mathrm{Cr}$ moment and the induced moment of the $s p$ atom decrease. In case II, the changes of different moments compensate resulting in the same integer total moment. The decrease of the Cr spin moment causes a small decrease of the width of the gap.

Next we used the frozen-magnon approach to calculate the exchange parameters for both systems and three values of the lattice constants (figure 21). The exchange interaction between the induced moments of the $s p$ atoms is very small and the corresponding parameters are not presented.

For the semiconductor lattice parameter (left panels in figure 2) the interactions are characterized by the large ferromagnetic coupling of the nearest $\mathrm{Cr}$ atoms. In $\mathrm{CrAs}$, this coupling is about twice the coupling in CrSe. In the zinc-blende structure the nearest surrounding of a $\mathrm{Cr}$ atom is formed by $4 \mathrm{sp}$ atoms. The second-nearest coordination sphere contains $\mathrm{Cr}$ atoms. Although the exchange interaction $\mathrm{Cr}-\mathrm{sp}$ atom is antiferromagnetic it makes the parallel directions of the $\mathrm{Cr}$ moments energetically preferable. Therefore the indirect exchange interaction of the $\mathrm{Cr}$ moments through 


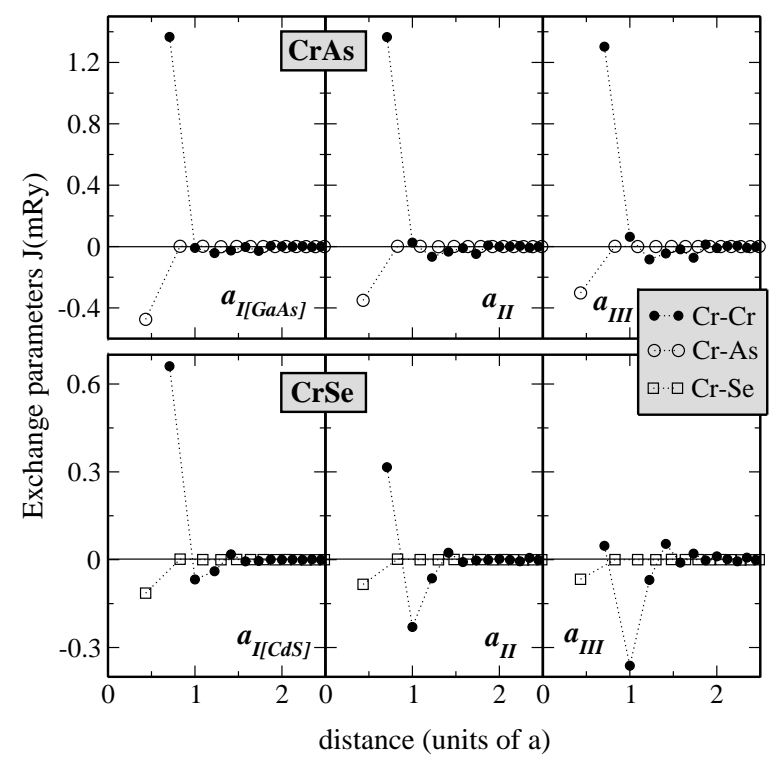

Figure 2. The exchange constants of $\mathrm{CrAs}$ (upper panel) and $\mathrm{CrSe}$ (lower panels) for three different lattice constants. The left panel corresponds to the experimental lattice constant of GaAs and CdS respectively for $\mathrm{CrAs}$ and $\mathrm{CrSe}$, the middle one to case II and the right one to case III.

the $s p$ atoms stabilizes further the ferromagnetic configuration of the $\mathrm{Cr}$ moments (a similar situation was found in full-Heusler alloys 61). The interaction is short range and involves only the nearest $\mathrm{Cr}$ atoms.

The influence of the lattice compression is very different for the two systems. In $\mathrm{CrAs}$, the compression leads to a small decrease of the exchange constants revealing the robustness of the ferromagnetism of the compound. On the other hand, in CrSe the effect of the compression is very strong. Already for the $\mathrm{a}_{I I}$ case where the system is still half-metallic the exchange interaction between the nearest $\mathrm{Cr}$ atoms is halved with respect to the case of the CdS lattice constant. The interaction between second-nearest $\mathrm{Cr}$ atoms strongly increases keeping antiferromagnetic character. This is reflected in a very small calculated Curie temperature (table 2). When the lattice is further compressed and the Fermi level moves into the minority-spin conduction band, the ferromagnetic interaction between the nearest $\mathrm{Cr}$ atoms almost vanishes whereas the antiferromagnetic interaction between the second nearest $\mathrm{Cr}$ atoms increases further. This leads to the instability of the ferromagnetic state.

The calculated Curie temperatures are collected in table 2. They reflect the properties of the exchange interactions discussed above. We report two estimations of the Curie temperature within the MFA, one with only the $\mathrm{Cr}-\mathrm{Cr}$ interactions taken into account and other with both $\mathrm{Cr}-\mathrm{Cr}$ and $\mathrm{Cr}-s p$ atom interactions considered, and one RPA estimation.

In case I, for both systems the Curie temperature is much higher than the room temperature. For CrAs, $T_{\mathrm{C}}$ is about twice larger than in CrSe and exceeds $1000 \mathrm{~K}$. The compression of the lattice leads in the case of CrAs to a very moderate decrease of the Curie temperature. In $\mathrm{CrSe}$, the $T_{\mathrm{C}}$ drops strongly with decreasing lattice 
Table 2. Calculated Curie temperatures for CrAs and CrSe. In the $3^{\text {rd }}$ and $4^{\text {th }}$ columns the MFA-estimations of $T_{\mathrm{C}}^{\mathrm{MFA}}$ are presented (respectively, with only the $\mathrm{Cr}-\mathrm{Cr}$ interactions and with both the $\mathrm{Cr}-\mathrm{Cr}$ and $\mathrm{Cr}-\mathrm{As}(\mathrm{Se})$ interactions taken into account. In the $5^{\text {th }}$ column, the RPA-estimation of $T_{\mathrm{C}}$ is presented. Negative values of $T_{\mathrm{C}}$ reveal the instability of the ferromagnetic structure.

\begin{tabular}{lc|ccc}
\hline Compound & $\mathrm{a}(\AA)$ & $T_{\mathrm{C}}^{\mathrm{MFA}}(\mathrm{Cr}-\mathrm{Cr})$ & $T_{\mathrm{C}}^{\mathrm{MFA}}$ & $T_{\mathrm{C}}^{\mathrm{RPA}}$ \\
\hline $\mathrm{CrAs}-\mathrm{a}_{I[G a A s]}$ & 5.65 & 1576 & 1600 & 1176 \\
$\mathrm{CrAs}-\mathrm{a}_{I I}$ & 5.53 & 1498 & 1512 & 1011 \\
$\mathrm{CrAs}-\mathrm{a}_{I I I}$ & 5.47 & 1376 & 1387 & 860 \\
\hline $\mathrm{CrSe}-\mathrm{a}_{I[C d S]}$ & 5.82 & 706 & 709 & 508 \\
$\mathrm{CrSe}-\mathrm{a}_{I I}$ & 5.62 & 109 & 117 & 24 \\
$\mathrm{CrSe}-\mathrm{a}_{I I I}$ & 5.56 & -248 & -251 & -194 \\
\hline
\end{tabular}

parameter becoming negative in case III revealing the instability of ferromagnetism.

Two mean-field estimations give very similar results (table2) revealing the leading role of the $\mathrm{Cr}-\mathrm{Cr}$ interactions. Therefore the neglect of the inter-sublattice Cr-sp atom interactions does not result in a substantial error for these compounds.

Since the intra-sublattice interactions dominate, the use of the RPA formula (7) is justified. Overall the $T_{\mathrm{C}}$ values within RPA are by $20-30 \%$ smaller than the MFA ones. The difference between MFA and RPA estimations is explained by a different weighting of the spin-wave excitations. In the MFA all excitations are taken with equal weight whereas in the RPA the weight decreases with increasing energy of the excitation 62, 63.

The RPA is expected to provide better estimation of the Curie temperature. In section 5 we will discuss the relation between the MFA and RPA values of $T_{\mathrm{C}}$ in more detail. The only experimental information about the magnetic transition temperature of the systems considered is that the Curie temperature of CrAs is well above $400 \mathrm{~K}$ 12 which is in good correlation with our estimations.

In the case of $\mathrm{CrAs}$ we can compare the calculated $T_{\mathrm{C}}$ values of $1600 \mathrm{~K}$ within MFA and $1176 \mathrm{~K}$ within RPA with the results of previous ab-initio studies. Sanyal and collaborators calculated the Curie temperature within MFA and found a value of 1320 $\mathrm{K}$ for the GaAs experimental lattice constant and $1100 \mathrm{~K}$ for the InAs experimental lattice constant 28. Their Monte Carlo simulations gave for the same lattice constants $980 \mathrm{~K}$ and $790 \mathrm{~K}$, respectively. Kübler used a Ginzburg-Landau approach with a simplified form of the dynamical susceptibility and a relaxation parameter fitted to reproduce the experimental Curie temperature of NiMnSb. His estimation of $T_{\mathrm{C}}$ of CrAs gives $1041 \mathrm{~K}$ [52]. Finally, Sakuma's mean-field calculation for the theoretical equilibrium lattice constant of $5.82 \AA$ gave $T_{\mathrm{C}}$ about $1400 \mathrm{~K}$ [51]. Summarizing, all calculations agree that the Curie temperature of CrAs should exceed or be about 1000 $\mathrm{K}$. A high $T_{\mathrm{C}}$ value that is stable with respect to the variation of the lattice constant makes CrAs an interesting candidate for spintronics applications.

\section{Mn-compounds: MnSi, MnGe and MnAs}

We proceed with the discussion of Mn compounds. Here we study MnSi and MnGe which are isoelectronic with $\mathrm{CrAs}$ and $\mathrm{MnAs}$ which is isoelectronic with $\mathrm{CrSe}$.

In table 3 we present the atom-resolved and total magnetic moments. MnAs with 
Table 3. Similar to table 1 for the Mn-based compounds.

\begin{tabular}{lc|ccccc}
\hline Compound & $\mathrm{a}(\AA)$ & $m^{\mathrm{Mn}}$ & $m^{\mathrm{Z}}$ & $m^{\text {Void1 }}$ & $m^{\text {Void2 }}$ & $m^{\text {Total }}$ \\
\hline $\mathrm{MnAs}-\mathrm{a}_{I[I n P]}$ & 5.87 & 4.16 & -0.26 & -0.01 & 0.10 & 4.00 \\
$\mathrm{MnAs}-\mathrm{a}_{I I}$ & 5.73 & 4.09 & -0.20 & -0.01 & 0.11 & 4.00 \\
$\mathrm{MnAs}-\mathrm{a}_{I I I}$ & 5.68 & 4.01 & -0.19 & -0.01 & 0.10 & 3.91 \\
\hline $\mathrm{MnGe}-\mathrm{a}_{I[G a A s]}$ & 5.65 & 3.43 & -0.39 & -0.08 & 0.04 & 3.00 \\
$\mathrm{MnGe}-\mathrm{a}_{I I}$ & 5.61 & 3.40 & -0.37 & -0.07 & 0.04 & 3.00 \\
$\mathrm{MnGe}-\mathrm{a}_{I I I}$ & 5.55 & 3.35 & -0.34 & -0.07 & 0.04 & 2.98 \\
\hline $\mathrm{MnSi}-\mathrm{a}_{I[G a A s]}$ & 5.65 & 3.51 & -0.49 & -0.07 & 0.05 & 3.00 \\
$\mathrm{MnSi}-\mathrm{a}_{I I}$ & 5.52 & 3.42 & -0.42 & -0.06 & 0.06 & 3.00 \\
$\mathrm{MnSi}-\mathrm{a}_{I I I}$ & 5.46 & 3.37 & -0.39 & -0.06 & 0.05 & 2.97 \\
\hline
\end{tabular}

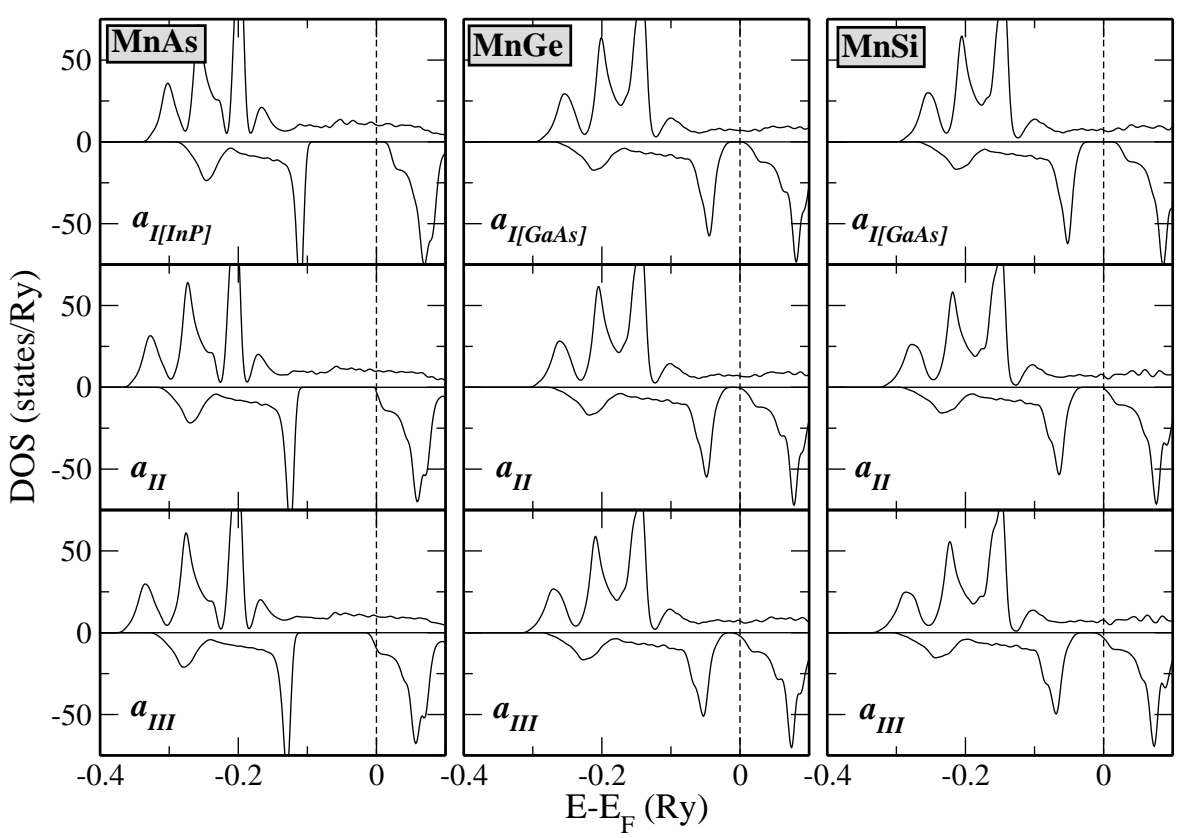

Figure 3. The density of states of MnAs, MnGe and MnSi. See the caption in figure 1 for the description of details.

the lattice constant of InP, and $\mathrm{MnSi}$ and MnGe with the lattice constant of GaAs are half-metallic and their total spin moments following the relation presented above are $4 \mu_{\mathrm{B}}$ for MnAs and $3 \mu_{\mathrm{B}}$ for $\mathrm{MnSi}$ and MnGe (see table 3 and figure 3). The Mn spin moment is about $3.5 \mu_{\mathrm{B}}$ for $\mathrm{MnSi}$ and $\mathrm{MnGe}$, and exceeds the $4 \mu_{\mathrm{B}}$ in the case of MnAs reflecting the presence of one more valence electron in the latter compound. The moments of the $s p$ atoms are antiferromagnetically coupled to the Mn moments. The value of the half-metallic gap is determined by the value of the exchange splitting and the energy position of the $p$ states of the $s p$ atom while the value of the exchange splitting is governed by the value of the Mn moment. As a result, the largest gap is obtained for MnAs followed by $\mathrm{MnSi}$ and the smallest gap is obtained for MnGe.

As for the $\mathrm{Cr}$ compounds the contraction of the lattice increases the energy of 


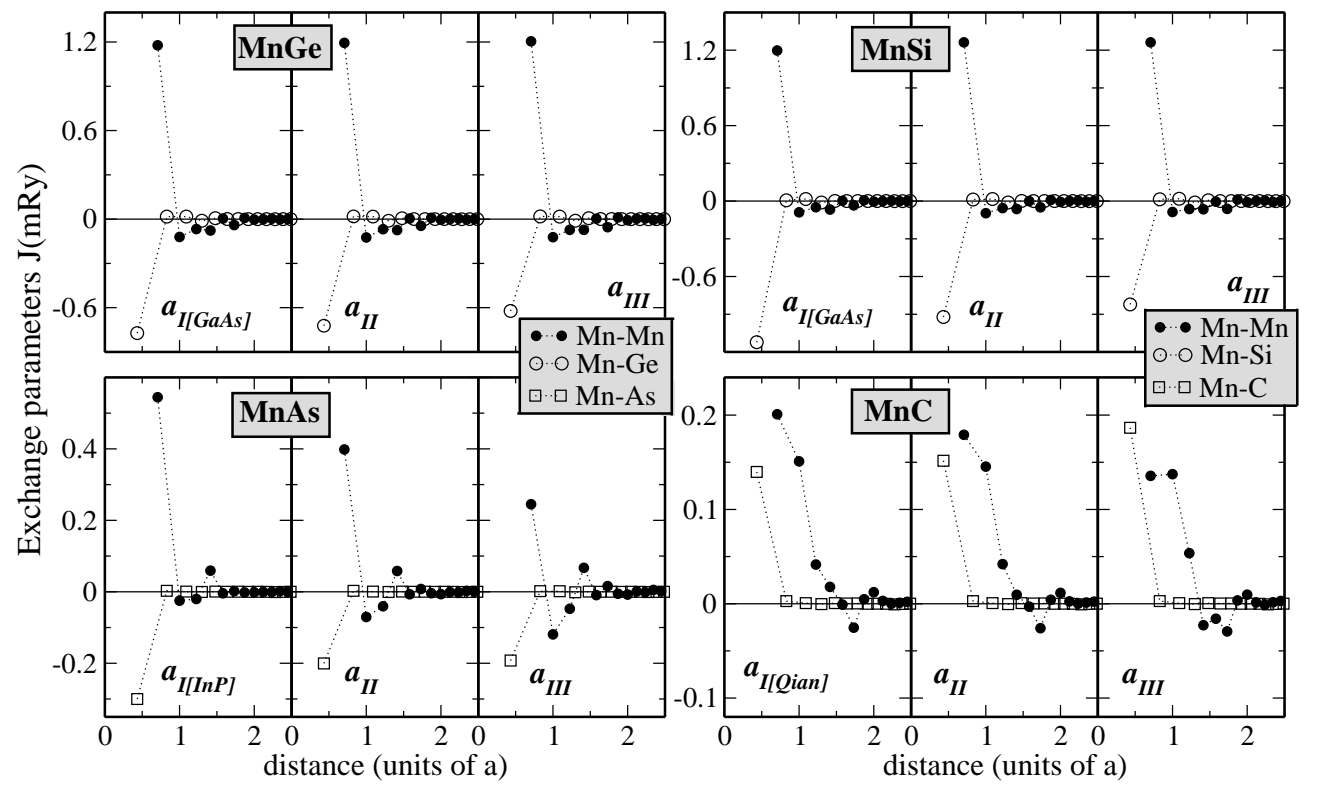

Figure 4. Variation of the exchange constants for the Mn-based compounds. Left panels correspond to the experimental lattice constants of semiconductors (see table 3), middle panel to case II and the right panel to case III.

the majority $p$ states leading to an increased Fermi level. For all three compounds the hybridization between the $p$ states of the $s p$ atom and the $t_{2 g}$ states of Mn increases and the Mn spin moment slightly decreases with the lattice contraction. The smaller Mn moments and, as a result, smaller exchange splittings lead to slightly smaller gap-widths.

In figure 4 we present the calculated exchange constants. The exchange interaction between the induced moments of $s p$ atoms is very weak and is not presented. There is strong similarity in the behavior of the exchange parameters for the $\mathrm{Mn}$ compounds considered here and the $\mathrm{Cr}$ compounds discussed in the preceding section. The properties of $\mathrm{MnGe}$ and $\mathrm{MnSi}$ are analogous to the properties of the isovalent $\mathrm{CrAs}$ while properties of MnAs are similar to the properties of isovalent $\mathrm{CrSe}$. Therefore the corresponding parts of the discussion of the $\mathrm{Cr}$ compounds remain valid also for the Mn systems.

Ferromagnetism is stabilized by both the direct Mn-Mn exchange interaction and by the indirect interaction through the $s p$ atoms. The exchange interactions are shortrange and the Mn-Mn exchange constants for MnAs are about a factor two smaller than for MnSi and MnGe.

The compression has little effect on MnSi and MnGe in contrast to MnAs. Again there is a strong similarity between $\mathrm{MnAs}$ and $\mathrm{CrSe}$ although the dependence of the exchange parameters on the lattice constant in MnAs is somewhat weaker. In particular, the antiferromagnetic exchange interaction between second-nearest $\mathrm{Mn}$ atoms developing with contraction has lower value than in CrSe. This results in preserving the ferromagnetic ground state of $\mathrm{MnAs}$ for all lattice parameters considered while in CrSe the ferromagnetic state becomes unstable (table 2). 
Table 4. Calculated spin moments for MnAs, MnGe and MnSi. See the caption in table 2 for the description.

\begin{tabular}{lc|ccc}
\hline Compound & $\mathrm{a}(\AA)$ & $T_{\mathrm{C}}^{\mathrm{MFA}}(\mathrm{Mn}-\mathrm{Mn})$ & $T_{\mathrm{C}}^{\mathrm{MFA}}$ & $T_{\mathrm{C}}^{\mathrm{RPA}}$ \\
\hline $\mathrm{MnAs}-\mathrm{a}_{I[I n P]}$ & 5.87 & 679 & 701 & 551 \\
$\mathrm{MnAs}-\mathrm{a}_{I I}$ & 5.73 & 400 & 417 & 275 \\
$\mathrm{MnAs}-\mathrm{a}_{I I I}$ & 5.68 & 201 & 230 & 136 \\
\hline $\mathrm{MnGe}-\mathrm{a}_{I[G a A s]}$ & 5.65 & 1160 & 1234 & 716 \\
$\mathrm{MnGe}-\mathrm{a}_{I I}$ & 5.61 & 1180 & 1241 & 740 \\
$\mathrm{MnGe}-\mathrm{a}_{I I I}$ & 5.55 & 1184 & 1231 & 695 \\
\hline $\mathrm{MnSi}-\mathrm{a}_{I[G a A s]}$ & 5.65 & 1255 & 1402 & 848 \\
$\mathrm{MnSi}-\mathrm{a}_{I I}$ & 5.52 & 1310 & 1401 & 857 \\
$\mathrm{MnSi}-\mathrm{a}_{I I I}$ & 5.46 & 1291 & 1367 & 911 \\
\hline
\end{tabular}

The Curie temperatures of the Mn compounds are collected in table 4 Again, as for $\mathrm{Cr}$ compounds, the $\mathrm{Mn}-\mathrm{Mn}$ interaction is dominant and the account for the exchange interaction Mn-sp atom influences the value of the Curie temperature weakly. In agreement with the properties of the exchange constants the Curie temperatures of MnGe and MnSi depend weakly on the lattice parameter in contrast to MnAs where this dependence is strong. The Curie temperature of MnAs at highest contraction considered assumes the value of about $\frac{1}{4}$ th of the Curie temperature at the semiconductor lattice constant. The RPA gives values of $T_{\mathrm{C}}$ that are about 30 $\%$ smaller than the corresponding values within the MFA. For MnSi and MnGe, also the RPA value of $T_{\mathrm{C}}$ remains considerably above the room temperature at all lattice constants studied. The stability of the high $T_{\mathrm{C}}$ value with respect to the variation of the lattice constant makes MnGe and MnSi promising candidates for spintronics applications. MnSi has an additional advantage of a larger half-metallic gap.

Next we compare the results of our calculation of $T_{\mathrm{C}}$ with the results of previous calculations where available. For MnAs, we can compare our MFA-estimation of 701 $\mathrm{K}$ and RPA estimation of $551 \mathrm{~K}$ obtained for the InP lattice constant with the Curie temperatures reported by Sanyal et al 28]. The MFA-estimation of Sanyal et al gave the value of $640 \mathrm{~K}$ for the InAs lattice parameter which is very close to the parameter used in our calculations. Their Monte Carlo simulation gave a $T_{\mathrm{C}}$ of $530 \mathrm{~K}$ which is very close to our RPA estimation. Kübler obtained the value of $671 \mathrm{~K}$ for MnAs with the InAs lattice constant. When he compressed the lattice down to the GaAs lattice constant of $5.65 \AA$ the $T_{\mathrm{C}}$ dropped sharply to $210 \mathrm{~K}[52$ in agreement with our results. Finally, Sakuma's MFA-estimation of the Curie temperature of MnGe for a lattice constant of $5.82 \AA$ gave a value of about $1200 \mathrm{~K}$ which is very close to our MFA value of $1234 \mathrm{~K}$ [51]. Thus the results of different calculations are in reasonable agreement with one another.

\section{5. $\mathrm{MnC}$}

The last Heusler compound studied in this paper is $\mathrm{MnC}$. This system was predicted to be half-metallic by Qian and collaborators [35]. First we discuss the results of the calculation with the theoretical equilibrium lattice constant reported by Qian et al. The magnetic properties of $\mathrm{MnC}$ differ from the properties of the $\mathrm{Mn}$ compounds discussed above. MnC has 11 valence electrons: 6 of majority-spin and 5 minority- 

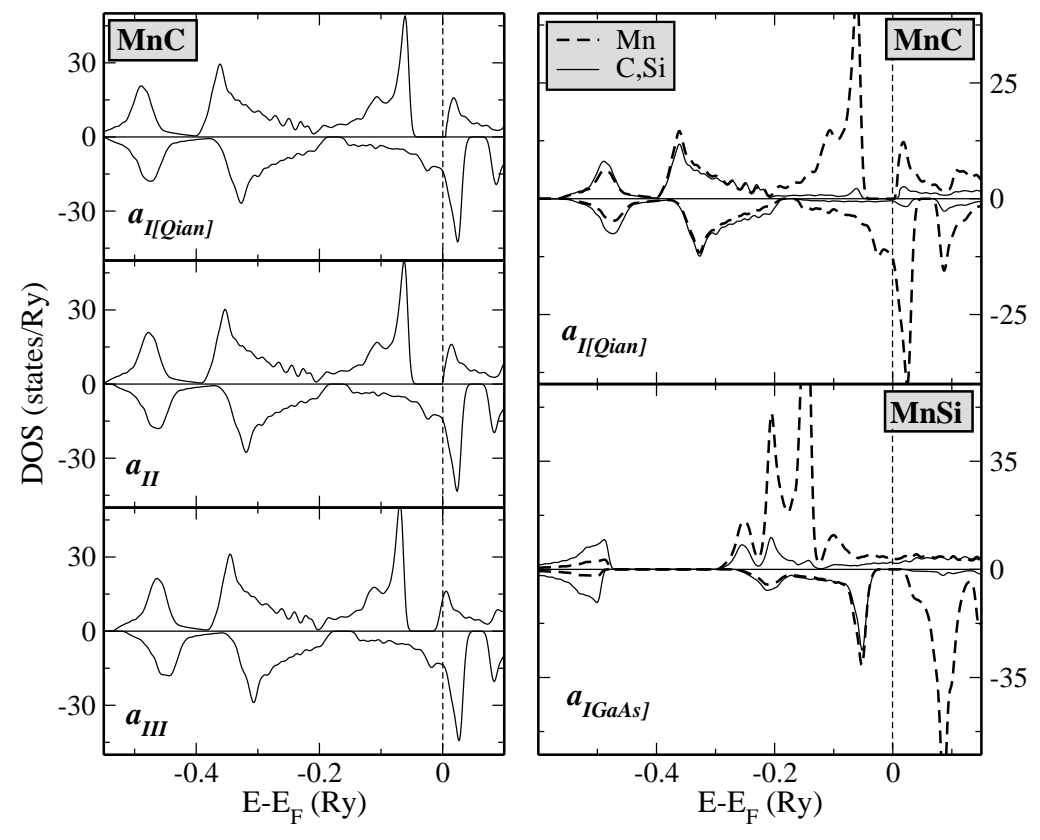

Figure 5. Left panel: calculated spin-resolved DOS of MnC. The upper panel corresponds to the lattice constant given in reference 35, the middle and lower panels to the $\mathrm{a}_{I I}$ and $\mathrm{a}_{I I I}$ cases in table 5 Right panel: Atom- and spin-resolved DOS for the half-metallic $\mathrm{MnC}$ and $\mathrm{MnSi}$ compounds.

spin electrons. This electron distribution results in a magnetic moment which is much smaller than in the compounds discussed above (table 5). In contrast to the other compounds the half-metallic gap is now in the majority-spin channel (figure 5). The majority-spin DOS has the following structure: low in energy in the interval [-0.55 Ry,$0.4 \mathrm{Ry}]$ lie $s$-states. Above, in the interval [-0.4 Ry,-0.2 Ry] there are three bonding hybrids between the $p$ states of $\mathrm{C}$ and the $t_{2 g}$ states of $\mathrm{Mn}$. This is seen in the atom-projected $\mathrm{DOS}$ of $\mathrm{MnC}$ (right panel in figure 5) where both $\mathrm{Mn}$ and $\mathrm{C}$ states have similar contribution in this energy interval. The group of states next in energy (interval $[-0.2 \mathrm{Ry},-0.05 \mathrm{Ry}]$ ) does not hybridize with the lower states. These are the $\mathrm{Mn} e_{g}$ states separated by a gap from the antibonding $\mathrm{C} p$-Mn $t_{2 g}$ hybrids. The Fermi level lies within this gap in the majority-spin DOS. For the minority-spin channel, the position of the Fermi level belongs to the region of the $e_{g}$ states. These states are shifted by the exchange splitting to higher energies with respect to the corresponding majority-spin states.

The comparison of the DOS of half-metallic $\mathrm{MnC}$ and $\mathrm{MnSi}$ (figure 5) allows to reveal the reason for the move of the semiconductor-type behavior from the spinminority channel in MnSi to the spin-majority channel in MnC. First, the two systems differ by the value of the magnetic moment that leads in the case of $\mathrm{MnC}$ to a smaller relative exchange shift of the spin-up and spin-down states and to the occupation of a larger number of the spin-down Mn $3 d$ states. In particular, the minority-spin $e_{g}$ states are partly occupied in $\mathrm{MnC}$ whereas in $\mathrm{MnSi}$ these states lie above the half-metallic gap. Second, there is difference in the position of the $e_{g}$ states in the hybridizational gap between bonding and antibonding $t_{2 g}$ - $p$ hybrids which in the case of MnSi results 
Table 5. Calculated spin magnetic moments and Curie temperatures for MnC. The value of $4.20 \AA$ is the equilibrium lattice constant calculated by Qian et al. 35].

\begin{tabular}{lc|ccccc}
\hline Compound & $\mathrm{a}(\AA)$ & $m^{\mathrm{Mn}}$ & $m^{\mathrm{Z}}$ & $m^{\text {Void1 }}$ & $m^{\text {Void2 }}$ & $m^{\text {Total }}$ \\
\hline $\mathrm{MnC}-\mathrm{a}_{I \text { (ref. [35] })}$ & 4.20 & 1.16 & -0.14 & -0.01 & -0.01 & 1.00 \\
$\mathrm{MnC}-\mathrm{a}_{I I}$ & 4.23 & 1.16 & -0.14 & -0.01 & -0.01 & 1.00 \\
$\mathrm{MnC}-\mathrm{a}_{I I I}$ & 4.27 & 1.13 & -0.16 & -0.01 & -0.01 & 1.05 \\
\hline Compound & $\mathrm{a}(\AA)$ & & $T_{\mathrm{C}}^{\mathrm{MFA}}(\mathrm{Mn}-\mathrm{Mn})$ & $T_{\mathrm{C}}^{\mathrm{MFA}}$ & $T_{\mathrm{C}}^{\mathrm{RPA}}$ \\
\hline $\mathrm{MnC}-\mathrm{a}_{I(\text { ref. }}$ [35] $)$ & 4.20 & & & 520 & 526 & 507 \\
$\mathrm{MnC}-\mathrm{a}_{I I}$ & 4.23 & & & 467 & 476 & 458 \\
$\mathrm{MnC}-\mathrm{a}_{I I I}$ & 4.27 & & & 346 & 363 & 327 \\
\hline
\end{tabular}

in the gap between occupied majority $e_{g}$ and empty antibonding hybrid states.

To extend the study of $\mathrm{MnC}$ to the states with the Fermi level lying at the top-edge of the half-metallic gap (case II) and above it (case III) we need in this case to expand the lattice (table 5) opposite to all systems considered above where the lattice must be compressed. This difference comes from the move of the semiconductor channel to the majority-spin subsystem. The expansion of the lattice makes the energy bands narrower and decreases the strength of the $3 d-p$ hybridization. At the same time the Mn moment increases increasing the exchange splitting between majority and minority states. These properties lead to increasing occupation of the majority-spin states and the loss of the half-metallicity (figure 5).

The calculated exchange constants are presented in figure 4 The ferromagnetism is stabilized by the direct $\mathrm{Mn}-\mathrm{Mn}$ interactions and the $\mathrm{Mn}-\mathrm{C}$ ferromagnetic coupling. Since the Mn moment is much smaller than in other compounds the value of the Mn$\mathrm{Mn}$ exchange interaction is substantially lower (figure 4). On the other hand, in $\mathrm{MnC}$ the first and second nearest Mn atoms are characterized by a similar strength of the ferromagnetic exchange interaction while in other systems only the nearest Mn atoms interact strongly. The expansion of the lattice leads to decreasing Mn-Mn exchange interactions.

The calculated Curie temperatures are presented in table [5] The Curie temperature of $\mathrm{MnC}$ is smaller than in other $\mathrm{Mn}$ compounds being about $500 \mathrm{~K}$ (it is comparable with the RPA estimation of $T_{\mathrm{C}}$ of $\mathrm{MnAs}$ ). The value of the Curie temperature decreases with expansion of the lattice showing again that half-metallicity is favorable for high Curie temperature.

A remarkable feature of the calculation for $\mathrm{MnC}$ is a very small difference between the $T_{\mathrm{C}}$ values estimated within the MFA and the RPA. In section 3 we briefly discussed the reason behind the different values of the Curie temperatures estimated within MFA and RPA. A striking contrast between $\mathrm{MnC}$ and the systems considered in the previous sections allows gaining a deeper insight into the physics of the formation of the Curie temperature. We will base our analysis on the comparison of two systems: $\mathrm{MnC}$ and MnSi. Characterizing the relative difference of the MFA and RPA values of the Curie temperature by the relation $\left[T_{\mathrm{C}}^{\mathrm{MFA}}-T_{\mathrm{C}}^{\mathrm{RPA}}\right] / T_{\mathrm{C}}^{\mathrm{RPA}}$ we get for $\mathrm{MnC}$ a small value of $2.5 \%$ compared to a large value of $47 \%$ in $\mathrm{MnSi}$.

In MFA, the Curie temperature is determined by an arithmetic average of the magnon energies while in RPA $T_{\mathrm{C}}$ is determined by the harmonic average of the same 


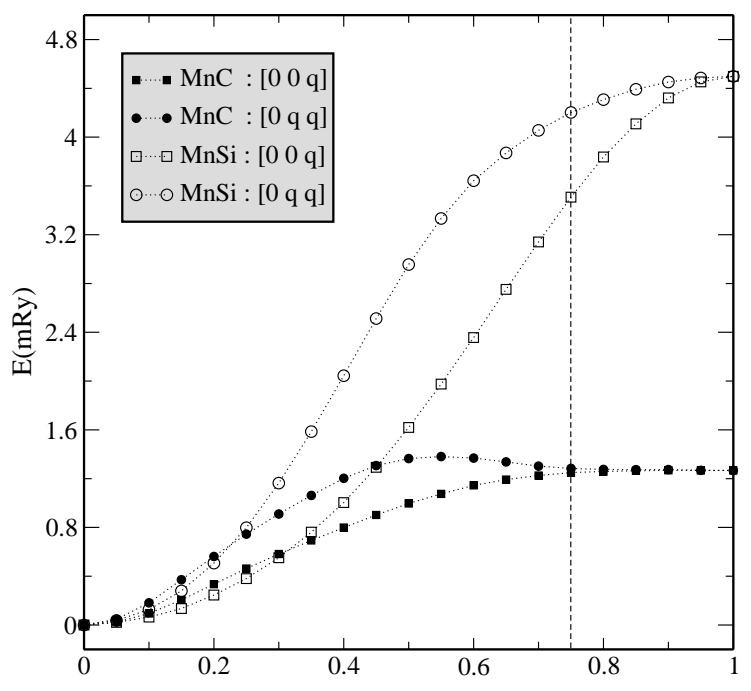

Figure 6. Frozen-magnon energies in $\mathrm{MnC}$ and $\mathrm{MnSi}$ for $\left[\begin{array}{lll}0 & 0 & \mathrm{q}\end{array}\right]$ and $\left[\begin{array}{lll}0 & \mathrm{q} & \mathrm{q}\end{array}\right]$ directions in the Brillouin zone. The vertical broken line shows the Brillouin zone boundary for the [0 q q] direction.

quantities. It is an arithmetic property that the MFA estimation is larger than the RPA one or equal to it if all averaged numbers are equal to each other. In terms of magnon energies, $T_{\mathrm{C}}^{\mathrm{MFA}}$ is equal to $T_{\mathrm{C}}^{\mathrm{RPA}}$ in the case that the magnon spectrum is dispersion-less: the magnon energy does not depend on the wave vector $\mathbf{q}$.

In figure [ 6 we plot the frozen-magnon energies of $\mathrm{MnSi}$ and $\mathrm{MnC}$ for two selected directions in the reciprocal space. We see that the $\mathrm{MnC}$ curves are flat in the second half of the $\mathbf{q}$ interval showing here a very weak dispersion. On the other hand, the MnSi curves are much closer to a simple cosinusoidal form with considerable dispersion in this part of the $\mathbf{q}$ interval. Comparing now the low-q parts of the curves we notice that $\mathrm{MnC}$ curves lie higher than the $\mathrm{MnSi}$ curves. Therefore, the relative contribution of the low-energy magnons to the RPA value of the Curie temperature is less important in $\mathrm{MnC}$ compared to $\mathrm{MnSi}$.

This combination of features of the wave-vector dependencies of the frozenmagnon energies is responsible for a much smaller difference between the RPA and MFA estimations of the Curie temperature in the two cases. Since the form of the magnon dispersion is reflected on the properties of the inter-atomic exchange interactions it is instructive to compare the patterns of the exchange interactions of the two systems. Taking into account that only Mn-Mn interactions contribute importantly in the Curie temperature we will focus on the comparison of these interactions.

The analysis of the Mn-Mn exchange interactions in MnSi and $\mathrm{MnC}$ (figure 4) shows a strong difference between the two systems. In MnSi, only the first-neighbor exchange interaction plays important role. All other interactions are much smaller. On the other hand, in the case of $\mathrm{MnC}$ the second-neighbor interaction is comparable with the first-neighbor one. The further few interactions though sizable play less important role. The presence of two first coordination spheres with similar values of the exchange interactions, on the one hand, makes the exchange interaction in $\mathrm{MnC}$ 
more long ranged and, on the other hand, it effectively increases the coordination number of the atoms with leading exchange interaction. Either of these properties leads to a better accuracy of the MFA estimation.

\section{Summary and Conclusions}

We have presented a detailed study of the stability of the ferromagnetism in six transition-metal compounds crystallizing in the zinc-blende structure. We calculated from first-principles the electronic structure and employed the frozen-magnon approximation to evaluate the exchange parameters and the Curie temperature. In the calculation of the Curie temperature we used both the mean-field and random-phase approximations.

Considering the CrAs and CrSe compounds we found in the case of CrAs a very high Curie temperature exceeding $1000 \mathrm{~K}$. The ferromagnetism of CrAs is stable with respect to the compression of the lattice. On the other hand CrSe possesses about two times lower Curie temperature which decreases strongly with lattice compression.

The properties of $\mathrm{MnSi}$ and MnGe are similar to the properties of the isovalent CrSe while the properties of MnAs are similar to the properties of isovalent CrSe.

$\mathrm{MnC}$ presents an interesting case that differs from all other systems studied. This system is also half-metallic with the half-metallic gap in the majority-spin channel in contrast to other systems where the half-metallic gap is in the minority-spin subsystem. The Curie temperature of $\mathrm{MnC}$ was found to be about $500 \mathrm{~K}$.

In all cases considered we found that half-metallicity is favorable for ferromagnetism and leads to an increased Curie temperature.

We conclude that two compounds, $\mathrm{MnSi}$ and $\mathrm{CrAs}$, possess properties which make them promising candidates for spintronics applications: large half-metallic gap and high Curie temperature. The stability of the Curie temperature in these systems allows to expect that being grown on the substrate of a binary semiconductor they will preserve the desired magnetic properties.

\section{Acknowledgments}

The financial support of Bundesministerium für Bildung und Forschung is acknowledged.

\section{References}

[1] Prinz G A 1998 Science 2821660

[2] de Boeck J, van Roy W, Das J, Motsnyi V, Liu Z, Lagae L, Boeve H, Dessein K and Borghs G 2002 Semicond. Sci. Technol. 17 342; de Boeck J, van Roy W, Motsnyi V, Liu Z, Dessein K and Borghs G 2002 Thin Solid Films 4123

[3] de Groot R A, Mueller F M, van Engen P G and Buschow K H J 1983 Phys. Rev. Lett. 502024

[4] Soulen Jr R J, Byers J M, Osofsky M S, Nadgorny B, Ambrose T, Cheng S F, Broussard P R, Tanaka C T, Nowak J, Moodera J S, Barry A and Coey J M D 1998 Science 28285

[5] Schwarz K J. Phys. F: Met. Phys. 198616 L211; Korotin M A, Anisimov V I, Khomskii D I and Sawatzky G A 1998 Phys. Rev. Lett. 804305

[6] Yanase A and Siratori K 1984 J. Phys. Soc. Jpn 53312

[7] Galanakis I, Dederichs P H and Papanikolaou N 2002 Phys. Rev. B 66134428

[8] Galanakis I, Dederichs P H and Papanikolaou N 2002 Phys. Rev. B 66174429

[9] Galanakis I 2004 J. Phys.: Condens. Matter 163089

[10] Akai H 1998 Phys. Rev. Lett. 813002

[11] Sandratskii L M and Bruno P 2002 Phys. Rev. B 66134435 
[12] Akinaga H, Manago T and Shirai M 2000 Japan. J. Appl. Phys. 39 L1118

[13] Shirai M 2001 Physica E 10143

[14] Shirai M 2003 J. Appl. Phys. 93 6844; Shirai M 2004 J. Phys.: Condens. Matter 16 S5525

[15] Mizuguchi M, Akinaga H, Manago T, Ono K, Oshima M and Shirai M 2002 J. Magn. Magn. Mater. 239269

[16] Zhao J H, Matsukura F, Takamura K, Abe E, Chiba D and Ohno H 2001 Appl. Phys. Lett. 79 2776

[17] Ono K, Okabayashi J, Mizuguchi M, Oshima M, Fujimori A and Akinaga H 2002 J. Appl. Phys. 918088

[18] Mizuguchi M, Akinaga H, Manago T, Ono K, Oshima M, Shirai M, Yuri M, Lin H J, Hsieh H $\mathrm{H}$ and Chen C T 2002 J. Appl. Phys. 917917

[19] Landolt-Börnstein, New Series, Group III, Vol. 27, Pt.a edited by K Adachi and S Ogawa (Springer-Verlag, Heidelberg) 1988

[20] Wei S H and Zunger A 1987 Phys. Rev. B 352340

[21] Sanvito S and Hill N A 2000 Phys. Rev. B 6215553

[22] Continenza A, Picozzi S, Geng W T and Freeman A J 2001 Phys. Rev. B 64085204

[23] Zhao Y -J, Geng W T, Freeman A J and Delley B 2002 Phys. Rev. B 65113202

[24] Xie W-H, Xu Y-Q, Liu B G and Pettifor D G 2003 Phys. Rev. Lett. 91037204

[25] Galanakis I and Mavropoulos Ph 2003 Phys. Rev. B 67104417

[26] Liu B G 2003 Phys. Rev. B 67172411

[27] Pask J E, Yang L H, Fong C Y, Pickett W E and Dag S 2003 Phys. Rev. B 67224420

[28] Sanyal B, Bergqvist L and Eriksson O 2003 Phys. Rev. B 68054417

[29] Xu Y G, Liu B G and Pettifor D G 2002 Phys. Rev. B 66184435

[30] Xie W -H and Liu B G 2003 J. Phys.: Condens. Matter 155085

[31] Yao K L, Gao G Y, Liu Z L and Zhu L 2005 Solid State Commun. 133301

[32] Zhang M, Hu H, Liu G, Y. Cui, Liu Z, Wang J, Wu G, Zhang X, Yan L, Liu H, Meng F, Qu J and Li Y 2003 J. Phys.: Condens. Matter 15 5017; Zhang M, Hu H, Liu G, Liu Z, Cui Y and Wu G 2004 J. Low Temp. Phys. 135 267; Zhang M, Hu H, Liu Z, Liu G, Cui Y and Wu G 2004 J. Magn. Magn. Mater. 27032

[33] Xie W -H, Liu B G and Pettifor D G 2003 Phys. Rev. B 68134407

[34] Zhang M, Brück E, de Boer F R, Liu G D, Hu H N, Liu Z H, Cui Y T and Wu G C $2004 J$. Mater. Res. 192738

[35] Qian M C, Fong C Y and Yang L H 2004 Phys. Rev. B 70052404

[36] Nakao M 2004 Phys. Rev. B 69214429

[37] Qian M C, Fong C Y, Pickett W E and Wang H Y 2004 J. Appl. Phys. 957459

[38] Shi L J and Liu B G 2005 J. Phys.: Condens. Matter 171209

[39] Yamana K, Geshi M, Tsukamoto H, Uchida I, Shirai M, Kusakabe K and Suzuki N $2004 \mathrm{~J}$. Phys.: Condens. Matter 16 S5815

[40] Zhao Y -J and Zunger A 2005 Phys. Rev. B in press; preprint arXiv:cond-mat/0408707

[41] Zheng J -C and Davenport J W 2004 Phys. Rev. B 69144415.

[42] Galanakis, I 2002 Phys. Rev. B 66012406

[43] Fong C Y, Qian M C, Pask J E, Yang L H and Dag S 2004 Appl. Phys. Lett. 84 239; Fong C Y and Qian MC 2004 J. Phys.: Condens. Matter 16 S5669.

[44] Mavropoulos Ph and Galanakis I 2004 J. Phys.: Condens. Matter 164261

[45] Nagao K, Shirai M and Miura Y 2004 J. Appl. Phys. 956518

[46] Geshi M, Kusakabe K and Suzuki N 2004 J. Phys.: Condens. Matter 16 S5701

[47] Qian M C, Fong C Y, Pickett W E, Pask J E, Yang L H and Dag S 2005 Phys. Rev. B 71012414

[48] Bengone O, Eriksson O, Fransson J, Turek I, Judrnovsky J and Drchal V 2004 Phys. Rev. B 70 035302

[49] Mavropoulos Ph, Sato K, Zeller R, Dederichs P H, Popescu V and Ebert H 2004 Phys. Rev. B 69054424

[50] Chioncel L, Katsnelson M I, de Wijs G A, de Groot R A and Lichtenstein A I 2005 Phys. Rev. B 71085111

[51] Sakuma A 2002 J. Phys. Soc. Japan 712534

[52] Kübler J 2003 Phys. Rev. B 67220403

[53] Williams A R, Kübler J and Gelatt C D 1979 Phys. Rev. B 196094

[54] Andersen O K 1975 Phys. Rev. B 123060

[55] Perdew J P and Wang Y 1992 Phys. Rev. B 4513244

[56] Şaşıg̃lu E, Sandratskii L M and Bruno P 2004 Phys. Rev. B 70024427

[57] Şaşıõlu E, Sandratskii L M and Bruno P 2005 J. Phys.: Condens. Matter 17995

[58] Rosengaard N M and Johansson B 1997 Phys. Rev. B 55 14975; Halilov S V, Eschrig H, Perlov 
A Ya and Oppeneer P M 1998 Phys. Rev. B 58 293; Sandratskii L M and Bruno P 2003 Phys. Rev. B 67214402

[59] Anderson P W, 1963, Theory of magnetic exchange interactions: Exchange in insulators and semiconductors, in Solid State Physics, edited by F. Seitz and Turnbull (Academic Press, New York), Vol. 14 pp. 99-214.

[60] Şaşıõlu E, Sandratskii L M and Bruno P 2005 J. Magn. Magn. Mater. 290-291 385.

[61] Kübler J, Williams A R and Sommers C B 1983 Phys. Rev. B 281745

[62] Pajda M, Kudrnovsky J, Turek I, Drchal V and Bruno P 2001 Phys. Rev. B 64174402

[63] Bouzerar G, Kudrnovský J, Bergqvist L, and Bruno P, 2003 Phys. Rev. B 68, 081203 